\title{
On the Derivation of the Schroedinger Equation in a Riemannian Manifold
}

\author{
K. R. Parthasarathy \\ Received January 17, 1968
}

\begin{abstract}
Under certain conditions it is shown that the kinetic part of the dynamical operator of a quantum mechanical system with a Riemannian manifold as configuration space is the Laplace-Beltrami operator.
\end{abstract}

\section{§1. Introduction}

In his book on the "Mathematical foundations of quantum mechanics" [2]. MACKEY raises the problem of characterising the kinetic part of the Schroedinger equation in a Riemannian manifold. The main aim of this paper is to show that under certain conditions the dynamical operator of a quantum mechanical system with a Riemannian manifold as its configuration space has its kinetic part locally unitarily equivalent to the Laplace-Beltrami operator. Since in a Riemannian manifold there need not exist one parameter groups of isometries it seems necessary to characterise the Schroedinger operator without using the notion of momentum. In the case of Euclidean configuration space MACKEY obtains the kinetic part of the Schroedinger operator by equating the velocity operator to a constant multiple of the momentum operator. Instead we start from the assumption that the acceleration operator is equal to a constant times the force operator.

In general notations and terminology we follow [2]. Regarding the basic properties of Riemannian manifolds and notations of tensor calculus we refer to [1].

\section{§ 2. Quantum Mechanical Systems with one Degree of Freedom}

Let $R$ denote the real line and $L_{2}(R)$ the space of all complex valued functions on $R$ square integrable with respect to the Lebesgue measure. For any complex valued function $g$ on $R$ we shall denote by $g^{(r)}$ the $r$-th. derivative of $g$. We shall adopt the notation $g$ for both the function $g$ as well as multiplication by $g$. For any two operators $A$ and $B$ of $L_{2}(R)$ into itself we shall denote by $[A, B]$ the operator $A B-B A$.

Let $H$ be the dynamical operator of a quantum mechanical system whose state vectors are unit vectors in $L_{2}(R)$. If $x$ denotes the position operator, then $i[H, x]$ is the velocity operator and $-[H,[H, x]]$ is the acceleration operator. 
We shall now derive the form of $H$ under the assumption that the acceleration operator is a multiplication operator and an energy equation is satisfied.

Theorem 2.1. Let $H$ be a symmetric differential operator with twice differentiable coefficients and

$$
\begin{aligned}
& m[H,[H, x]]=-v^{(1)} \\
& m\left[H,[H, x]^{2}\right]=c[H, v],
\end{aligned}
$$

where $m$ and $c$ are non zero constants and $v$ is an infinitely differentiable function. Suppose $v^{(3)}$ does not vanish on a set of positive Lebesgue measure. Then $c=-2$ and $H$ is a second order differential operator which is unitarily equivalent to an operator of the form $h \frac{d^{2}}{d x^{2}}+\frac{v}{2 h m}+\alpha$ where $h$ and $\alpha$ are constants. The unitary equivalence can be effected through a multiplication operator.

Proof. Condition b) implies that

$$
m[H, x][H,[H, x]]+m[H,[H, x]][H, x]=c[H, v] .
$$

By condition a) we have

Suppose

$$
[H, x] v^{(1)}+v^{(1)}[H, x]=-c[H, v] .
$$

$$
H=a_{n} \frac{d^{n}}{d x^{n}}+a_{n-1} \frac{d^{n-1}}{d x^{n-1}}+\cdots+a_{0}
$$

where $a_{n} \neq 0$. By applying the operators on either side of (2.1) to $C^{\infty}$ functions with compact supports and equating the coefficients of $\frac{d^{k}}{d x^{k}}$, $0 \leqq k \leqq n-1$, we have

$$
(k+1) a_{k+1} v^{(1)}+\sum_{r=k+1}^{n} r a_{r}\left(\begin{array}{c}
r-1 \\
k
\end{array}\right) v^{(r-k)}=-c \sum_{r=k+1}^{n} a_{r}\left(\begin{array}{l}
r \\
k
\end{array}\right) v^{(r-k)} .
$$

Putting $k=n-1$, we get

$$
2 n a_{n} v^{(1)}=-c n a_{n} v^{(1)} .
$$

Since $v^{(1)} \neq 0, c=-2$. Putting $k=n-3$, we have

$$
\left(\begin{array}{l}
n \\
3
\end{array}\right) a_{n} v^{(3)}=0 \text {. }
$$

Since $v^{(3)} \neq 0$ and $a_{n} \neq 0$, we have $\left(\begin{array}{l}n \\ 3\end{array}\right)=0$. I.e., $n \leqq 2$. In other words $H$ is a second order differential operator.

Suppose

$$
H=a \frac{d^{2}}{d x^{2}}+b \frac{d}{d x}+d .
$$

Substituting this operator in condition $(a)$, we obtain $m\left\{2 a a^{(1)} \frac{d^{2}}{d x^{2}}+\left(2 a a^{(2)}+2 b a^{(1)}\right) \frac{d}{d x}+a b^{(2)}+b b^{(1)}-2 a d^{(1)}\right\}=-v^{(1)}$. 
Thus $a a^{(1)}=0$. In other words $a=h$ where $h$ is a constant.

Further

Hence

$$
m\left(h b^{(2)}+b b^{(1)}-2 h d^{(1)}\right)=-v^{(1)} .
$$

$$
d=\frac{1}{2 h}\left(h b^{(1)}+b^{2} / 2+v / m\right)+\alpha
$$

where $\alpha$ is a constant. Thus

$$
H=h \frac{d^{2}}{d x^{2}}+v / 2 h m+b \frac{d}{d x}+\frac{1}{2 h}\left(h b^{(1)}+b^{2} / 2\right)+\alpha .
$$

The symmetry of $H$ implies that $b$ is purely imaginary. Consider the unitary operator $U$ defined by

$$
U f=f \exp \left[-\frac{1}{2 h} \int_{0}^{x} b(t) d t\right] .
$$

A simple calculation shows that

$$
U^{-1} H U=h \frac{d^{2}}{d x^{2}}+\frac{v}{2 h m}+\alpha .
$$

This completes the proof.

Remark. Theorem 2.1 shows that one may take $\frac{h}{2 m} \frac{d^{2}}{d x^{2}}+\frac{v}{h}$ as the most general dynamical operator of a quantum mechanical system with one degree of freedom. Condition $(b)$ of the theorem can be written as

$$
\left[H,-\frac{m}{2}[H, x]^{2}-v\right]=0
$$

$-[H, x]^{2}$ is the square of the velocity operator and $-v$ is the potential energy operator.

\section{§ 3. Systems with $n$ Degrees of Freedom}

We shall now consider a quantum mechanical system whose configuration space is the $n$-dimensional real Euclidean space $R^{n}$. Let $H$ be the dynamical operator of the system acting in the Hilbert space $L_{2}\left(R^{n}\right)$ of complex valued functions square integrable with respect to the Lebesgue measure. In the preceding section we derived the form of $H$ under the assumption that an energy equation is satisfied and the acceleration operator is a multiplication operator. We shall now replace the energy equation by the assumption that $H$ is a second order differential operator. For any twice differentiable function $\varphi$ we assume that $[[H, \varphi], \varphi]=0$ if and only if $\varphi$ is a constant. This assumption simply means that if a function of the position coordinates can be observed simultaneously with its rate of change, then it is a constant. We now have the following theorem. 
Theorem 3.1. Let $H$ be a symmetric second order differential operator of the form

$$
H=\sum_{i, j} a_{i j} \frac{\partial^{2}}{\partial x_{i} \partial x_{j}}+\sum_{i} b_{i} \frac{\partial}{\partial x_{i}}+c
$$

where $a_{i j}, b_{i}$ and $c$ are twice differentiable. Suppose for any twice differentiable function $\varphi$, the equation $[[H, \varphi], \varphi]=0$ holds if and only if $\varphi$ is a constant.

Let

$$
m_{i}\left[H,\left[H, x_{i}\right]\right]=-V_{i}, \quad i=1,2 \ldots n
$$

where $V_{i}$ are once differentiable functions and $m_{i}$ are constants. Then $H$ is unitarily equivalent to an operator of the form

$$
\sum_{i, j} a_{i j} \frac{\partial^{2}}{\partial x_{i} \partial x_{j}}+v
$$

where $\left(\left(a_{i j}\right)\right)$ is a constant non singular positive or negative definite matrix such that

$$
A^{-1} M^{-1} J=J^{\prime} M^{-1} A^{-1} .
$$

Here

$$
A=\left(\left(a_{i j}\right)\right), M=\left(\left(m_{i} \delta_{i j}\right)\right), J=\left(\left(\frac{\partial V_{i}}{\partial x_{j}}\right)\right),
$$

$J^{\prime}$ is the transpose of $J$ and $v$ is a function satisfying the equations

$$
\sum_{j} a_{i j} \frac{\partial v}{\partial x_{j}}=\frac{V_{i}}{2 m_{i}} .
$$

The unitary equivalence here can be effected by a multiplication operator.

Conversely any operator of the form described above satisfies Eq. (3.1).

Remark 1. If there exists a function $V$ such that $V_{i}=\frac{\partial V}{\partial x_{i}}$ for all $i$, then (3.2) and (3.3) are automatically satisfied if $A=\left(\left(-\frac{h}{2 m_{i}} \delta_{i j}\right)\right)$ and $v=V$ where $h$ is a constant. $H$ then assumes the standard form

$$
-\sum_{i} \frac{h}{2 m_{i}} \frac{\partial^{2}}{\partial x_{i}^{2}}+\frac{V}{h} \text {. }
$$

Proof of Theorem 3.1. First of all we observe that without loss of generality we may assume that $a_{i j}=a_{j i}$ for all $i$ and $j$. The symmetry of $H$ implies that the $a_{i j}$ are all real. Since under coordinate transformations the $a_{i j}$ behave like the coefficients of a second order symmetric contravariant tensor we may and do employ the standard notations of tensor calculus. In particular repeated index in a product implies that summation has been carried out. We shall denote $\frac{\partial a_{i j}}{\partial x_{k}} \frac{\partial^{2} a_{i j}}{\partial x_{k} \partial x_{l}} \ldots$ by 
$a_{i j, k} a_{i j, k l} \ldots$ respectively. Simple calculations show that

$$
\begin{aligned}
{\left[H, x_{i}\right]=} & 2 a_{i j} \frac{\partial}{\partial x_{j}}+b_{i} \\
{\left[H,\left[H, x_{i}\right]\right]=} & 2 a_{k l}\left(a_{i r, k} \frac{\partial^{2}}{\partial x_{l} \partial x_{r}}+a_{i r, l} \frac{\partial^{2}}{\partial x_{k} \partial x_{r}}\right)-2 a_{i r} a_{k l, r} \frac{\partial^{2}}{\partial x_{k} \partial x_{l}} \\
& +2 a_{k l} a_{i r, k l} \frac{\partial}{\partial x_{r}}+a_{k l}\left(\frac{\partial b_{i}}{\partial x_{l}} \frac{\partial}{\partial x_{k}}+\frac{\partial b_{i}}{\partial x_{k}} \frac{\partial}{\partial x_{l}}\right) \\
& +2\left(b_{l} a_{i r, l} \frac{\partial}{\partial x_{r}}-a_{i r} \frac{\partial b_{l}}{\partial x_{r}} \frac{\partial}{\partial x_{l}}\right)+a_{k l} \frac{\partial^{2} b_{i}}{\partial x_{k} \partial x_{l}} \\
& +b_{l} \frac{\partial b_{i}}{\partial x_{l}}-2 a_{i r} \frac{\partial c}{\partial x_{r}}
\end{aligned}
$$

By (3.1), the coefficients of $\frac{\partial^{2}}{\partial x_{k} \partial x_{l}}$ and $\frac{\partial}{\partial x_{k}}$ in (3.4) must vanish. Hence

$$
a_{r l} a_{i k, r}+a_{k r} a_{i l, r}-a_{i r} a_{k l, r}=0 \quad \text { for every } i, k, l .
$$

Interchanging $i$ and $k$ in (3.5) and adding to (3.5) we obtain

$$
a_{r l} a_{i k, r}=0 \quad \text { for every } i, k, l \text {. }
$$

If we put $\varphi=a_{i k}$ it follows from (3.6) that

$$
[[H, \varphi], \varphi]=2 a_{r l} \frac{\partial \varphi}{\partial x_{r}} \frac{\partial \varphi}{\partial x_{l}}=0 .
$$

Thus by hypothesis $\varphi$ is a constant. In other words all the $a_{i j}$ are constant. Let $\psi$ by the linear function $c_{i} x_{i}$ where $c_{1}, c_{2}, \ldots, c_{n}$ are real constants. Then

$$
[[H, \psi], \psi]=2 a_{i j} c_{i} c_{j} .
$$

Hence the quadratic form $a_{i j} c_{i} c_{j}=0$ if and only if all the $c_{i}$ 's are zero. Thus the constant matrix $\left(\left(a_{i j}\right)\right)$ is non singular and positive or negative definite. Hence its inverse exists and we shall denote it by $\left(\left(a^{i j}\right)\right)$.

Equating the coefficient of $\frac{\partial}{\partial x_{k}}$ in (3.4) to zero we have

$$
a_{k l} \frac{\partial b_{i}}{\partial x_{l}}-a_{i l} \frac{\partial b_{k}}{\partial x_{l}}=0 \quad \text { for every } i, k .
$$

Putting $b^{i}=a^{i}{ }^{j} b_{j}$, we can rewrite (3.7) as

$$
\frac{\partial b^{i}}{\partial x_{j}}=\frac{\partial b^{j}}{\partial x_{i}} \quad \text { for every } i, j .
$$

Hence there exists a function $B$ such that

In other words

$$
b^{i}=\frac{\partial B}{\partial x_{i}} .
$$

$$
b_{i}=a_{i j} \frac{\partial B}{\partial x_{j}} \quad \text { for every } i
$$


Thus $H$ is of the form

$$
a_{i j} \frac{\partial^{2}}{\partial x_{i} \partial x_{j}}+a_{i j} \frac{\partial B}{\partial x_{j}} \frac{\partial}{\partial x_{i}}+c
$$

where $a_{i j}$ is a non singular positive or negative definite constant matrix. Since $H$ is symmetric, $B$ is purely imaginary. Let $U$ be the unitary operator defined by

$$
U f=f \exp -\frac{B}{2}
$$

Then

$$
H^{\prime}=U^{-1} H U=a_{i j} \frac{\partial^{2}}{\partial x_{i} \partial x_{j}}+v,
$$

where $v$ is some real valued function. Further $H^{\prime}$ satisfies (3.1). Hence substituting $H^{\prime}$ in (3.1) and (3.4) and equating constant terms we get

$$
a_{i j} \frac{\partial v}{\partial x_{j}}=\frac{V_{i}}{2 m_{i}} \quad \text { for all } i \text {. }
$$

In order that such a $v$ may exist it is necessary and sufficient that

$$
A^{-1} M^{-1} J=J^{\prime} M^{-1} A^{-1},
$$

where $A, M, J, J^{\prime}$ are the matrices described in the statement of the theorem. This completes the proof.

\section{\$4. Quantum Mechanical Systems on a Riemannian Manifold}

Let $H$ be the dynamical operator of a quantum mechanical system whose configuration space is a connected $C^{\infty}$ Riemannian manifold $M$ of dimension $n$ (the obvious modifications needed for the $C^{2}$ manifold can be made by the reader). We shall suppose that $H$ is a second order symmetric differential operator acting in the Hilbert space $L_{2}(\mu)$ of complex valued functions square integrable with respect to the Riemannian measure $\mu$.

Let $U$ be a fixed coordinate neighbourhood. We shall denote by $L_{2}(\mu, U)$ and $L_{2}(U)$ respectively the Hilbert spaces of complex valued functions on $U$ square integrable with respect to the restriction of $\mu$ to $U$ and the Lebesgue measure in $U$. Then there is a canonical isomorphism between $L_{2}(\mu, U)$ and $L_{2}(U)$ through a multiplication operator. We shall denote by $H_{U}$ the restriction of $H$ to $U . H_{U}$ can be considered as an operator in $L_{2}(\mu, U)$. The isomorphism between $L_{2}(\mu, U)$ and $L_{2}(U)$ takes the operator $H_{U}$ to an operator $H_{U}^{\prime}$ in $L_{2}(U)$. $H_{U}^{\prime}$ is a second order symmetric differential operator in $L_{2}(U)$.

In the preceding section we derived the form of $H$ under the assumption that the acceleration operators of the individual position coordinates are multiplication operators. In the case of a Riemannian manifold we have to replace the acceleration operators by slightly different ones since the connection coefficients enter the geodesic equations. 
Suppose $g_{i j}$ are the coefficients of the Riemannian metric, $\Gamma_{i j}^{k}$ the connection coefficients and $R_{j k l}^{i}$ the components of the curvature tensor derived from the metric in the neighbourhood $U$. We shall always assume that whenever there is a repeated index in any product expression summation has been carried over it. Any geodesic in $U$ is a solution of the differential equations

$$
\ddot{x}_{k}+\dot{x}_{i} \Gamma_{i j}^{k} \dot{x}_{j}=0, \quad k=1,2, \ldots, n .
$$

Hence we shall assume that the operators $H_{U}^{\prime}$ satisfy the condition that

$$
\left[H_{U}^{\prime},\left[H_{U}^{\prime}, x_{k}\right]\right]+\left[H_{U}^{\prime}, x_{i}\right] \Gamma_{i j}^{k_{j}}\left[H_{U}^{\prime}, x_{j}\right]
$$

is a multiplication operator for every $k$.

Before stating our main result we shall introduce a notation. Consider $R_{i j k l}$ obtained by lowering the index $i$ in the component $R_{j k l}^{i}$ of the curvature tensor. For the antisymmetric pairs of indices $i j(i<j)$ and $k l(k<l)$ in $R_{i j k l}$ introduce the labels $I$ and $J$. Then $\left(\left(R_{I J}\right)\right)$ is a matrix of order $\frac{n(n-1)}{2}$. Let

$$
C_{U}=\operatorname{det}\left(\left(R_{I J}\right)\right)
$$

We now have the following theorem.

Theorem 4.1. Let $H$ be a second order symmetric differential operator in $L_{2}(\mu)$. Let $U$ be a simply connected coordinate neighbourhood such that $C_{U} \neq 0$ for every point in $U$ and

$$
H_{U}^{\prime}=a_{i j} \frac{\partial^{2}}{\partial x_{i} \partial x_{j}}+b_{i} \frac{\partial}{\partial x_{i}}+c
$$

where $\left(\left(a_{i j}\right)\right)$ is nonsingular in $U, a_{i j}, b_{i}$ and $c$ are $C^{\infty}$ functions. Suppose

$$
\left[H_{U}^{\prime},\left[H_{U}^{\prime}, x_{k}\right]\right]+\left[H_{U}^{\prime}, x_{i}\right] \Gamma_{i j}^{k}\left[H_{U}^{\prime}, x_{j}\right]=V_{k} \text { for every } k
$$

where $V_{k}$ are $C^{\infty}$ functions. Then $H_{U}^{\prime}$ is unitarily equivalent to an operator of the form $h \Delta+V$ in $L_{2}(\mu, U)$ where $\Delta$ is the Laplace-Beltrami operator, $h$ is a constant and $V$ is a $C^{\infty}$ function. The unitary equivalence can be established through a multiplication operator.

Conversely any operator of the form $h \Delta+V$ as described above always satisfies Eq. (4.1) if we choose $V_{k}$ appropriately.

Remark. In the case when the manifold has dimension 2 the matrix $\left(\left(R_{I J}\right)\right)$ is of order one and $C_{U}$ is just the Gaussian curvature. The condition of the theorem thus reduces to the manifold possessing non zero curvature at every point in $U$.

If $G$ is the group of all complex $n \times n$ matrices of determinant one, $n>2$ and $K$ is the subgroup of unitary matrices then $G / K$ is a symmetric space which is an analytic Riemannian manifold with $C_{U} \equiv 0$ for every coordinate neighbourhood $U$. 
Proof of Theorem 4.1. The operator $\left[H_{U}^{\prime},\left[H_{U}^{\prime}, x_{i}\right]\right]$ is of the form given by (3.4). We adopt the same notations as in $\S 3$. An easy calculation shows that

$$
\begin{aligned}
{\left[H_{U}^{\prime}, x_{i}\right] \Gamma_{i j}^{t}[} & \left.H_{U}^{\prime}, x_{j}\right]=4 \Gamma_{i j}^{t} a_{i k} a_{j l} \frac{\partial^{2}}{\partial x_{k} \partial x_{l}} \\
& +\left(4 a_{i k} \Gamma_{i j, k}^{t} a_{j l} \frac{\partial}{\partial x_{l}}+a_{i k} \Gamma_{i j}^{t} a_{j l, k} \frac{\partial}{\partial x_{l}}\right)+4 a_{i k} \Gamma_{i j}^{t} b_{j} \frac{\partial}{\partial x_{k}} \\
& +\Gamma_{i j}^{t} b_{i} b_{j}+2 a_{i k} \Gamma_{i j, k}^{t} b_{j}+2 a_{i k} \Gamma_{i j}^{t} \frac{\partial b_{j}}{\partial x_{k}}
\end{aligned}
$$

where $\Gamma_{i j, k}^{t}$ denotes $\frac{\partial \Gamma_{i j}^{t}}{\partial x_{k}}$. From (3.4), (4.1) and (4.2) we have

$$
a_{k r} a_{i l, r}-a_{i r} a_{k l, r}+a_{r l} a_{i k, r}+2 \Gamma_{r s}^{i} a_{r k} a_{s l}=0
$$

for every $i, k, l$. Interchanging $i$ and $k$ in the above equation and adding to same we obtain

$$
a_{s l}\left\{a_{i k, s}+\Gamma_{r s}^{i} a_{r k}+\Gamma_{r s}^{k} a_{r i}\right\}=0
$$

for every $i, k, l$. Since $\left(\left(a_{i j}\right)\right)$ is non singular in $U,(4.4)$ implies

$$
a_{i k, s}+\Gamma_{r s}^{i} a_{r l i}+\Gamma_{r s}^{k} a_{r i}=0
$$

for every $i, k, s$. This simply means that the covariant derivative of the second order symmetric tensor with components $a_{i j}$ vanishes identically in $U$.

The integrability condition for the Eq. (4.5), i.e.,

$$
\frac{\partial}{\partial x_{t}}\left(\Gamma_{r s}^{i} a_{r k}+\Gamma_{r s}^{k} a_{r i}\right)=\frac{\partial}{\partial x_{s}}\left(\Gamma_{r t}^{i} a_{r k}+\Gamma_{r t}^{k} a_{r i}\right)
$$

can be rewritten as

$$
\left(\tilde{a}_{i m} g_{j n}-\tilde{a}_{n j} g_{i m}\right) R_{k l}^{n m_{k l}}=0
$$

where $\tilde{a}_{i j}$ are the components of the matrix inverse to $\left(\left(a_{i j}\right)\right)$. Since $C_{U} \neq 0$, the matrix $\left(\left(R_{I J}\right)\right)$ is non singular and hence $\left(\left(R_{J}^{I}\right)\right)$ is non singular. Thus (4.6) holds if and only if

$$
\tilde{a}_{i j}=\varrho g_{i j} \quad \text { for all } i, j
$$

where $\varrho$ is a function. Hence $a_{i j}=\varrho^{-1} g^{i j}$. If we substitute $g^{i j}$ for $a_{i j}$, (4.5) is automatically satisfied. By substituting $\varrho^{-1} g^{i j}$ in (4.5) we obtain $\frac{\partial \varrho^{-1}}{\partial x_{s}}=0$ for all $s$. Thus $\varrho^{-1}$ is a constant. In other words there exists a constant $h$ such that

$$
a_{i j}=h g^{i j} \quad \text { for all } i, j .
$$

Using Eq. (3.4) (with $H=H_{U}^{\prime}$ ), (4.2) and (4.7) and equating the coefficient of $\frac{\partial}{\partial x_{u}}$ in the left hand side of (4.1) to zero, we get

$$
\begin{aligned}
h g^{k l} \frac{\partial^{2} g^{i u}}{\partial x_{k} \partial x_{l}} & +\left(g^{u l} \frac{\partial b_{l}}{\partial x_{l}}+b_{l} \frac{\partial g^{i u}}{\partial x_{l}}-g^{t l} \frac{\partial b_{u}}{\partial x_{l}}\right) \\
& +2 h\left(g^{i k} \Gamma_{i j, k}^{t} g^{j u}+g^{i k} \Gamma_{i j}^{t} \frac{\partial g^{j u}}{\partial x_{k}}\right)+2 g^{i u} \Gamma_{i j}^{t} b_{j}=0
\end{aligned}
$$


for all $t, u$. Interchanging $t$ and $u$ in (4.8), adding to the same and making use of (4.5) we obtain simply $0=0$.

We shall denote the vector field $g^{u} l \frac{\partial}{\partial x_{l}}$. by $X_{u}$. Interchanging $t$ and $u$ in (4.8) and subtracting from the same we obtain

$$
\begin{aligned}
\left(X_{u} b_{t}-X_{t} b_{u}\right) & +b_{j}\left(g^{t s} X_{u} g_{j s}-g^{u s} X_{t} g_{j s}\right) \\
& +h X_{j}\left(g^{t s} X_{u} g_{j s}-g^{u s} X_{t} g_{j s}\right)=0 \quad \text { for every } t, u .
\end{aligned}
$$

Writing $c_{s}=b_{j} g_{j s}$ and making use of the standard properties of vector fields, (4.9) can be rewritten as

$$
\left(g^{t s} X_{u}-g^{u s} X_{t}\right)\left(c_{s}+h X_{j} g_{j s}\right)=0
$$

for all $t, u$. Multiplying the left hand side of (4.10) by $g_{l c t} g_{l u}$ and adding over all $t$ and $u$, we obtain

$$
\frac{\partial}{\partial x_{l}}\left(c_{k}+h X_{j} g_{j k}\right)=\frac{\partial}{\partial x_{k}}\left(c_{l}+h X_{j} g_{j l}\right)
$$

for all $k$ and $l$. Since $U$ is simply connected there exists a $C^{\infty}$ function $d$ on $U$ such that

Hence

$$
\frac{\partial d}{\partial x_{k}}=c_{k}+h X_{j} g_{j k} \quad \text { for all } k
$$

$$
b_{t}=g^{t s} c_{s}=X_{t} d+h \frac{\partial g^{\ell r}}{\partial x_{r}}=X_{t} d+h \operatorname{div}_{L} X_{t},
$$

where $\operatorname{div}_{L}$ denotes the divergence with respect to the Lebesgue measure. Thus

$$
H_{U}^{\prime}=h\left(g^{i j} \frac{\partial^{2}}{\partial x_{i} \partial x_{j}}+\operatorname{div}_{L} X_{i} \frac{\partial}{\partial x_{i}}\right)+\left(X_{i} d\right) \frac{\partial}{\partial x_{i}}+c .
$$

Since $H_{U}^{\prime}$ and $g^{i j} \frac{\partial^{2}}{\partial x_{i} \partial x_{j}}+\operatorname{div}_{L} X_{i} \frac{\partial}{\partial x_{i}}$ are symmetric operators in $L_{2}(U)$ it follows that $d$ must be purely imaginary. Consider the unitary operator $W$ of $L_{2}(U)$ into itself defined by

Then

$$
W f=f \exp -\frac{d}{2 h} \text {. }
$$

$$
W^{-1} H_{U}^{\prime} W=h\left(g^{i j} \frac{\partial^{2}}{\partial x_{i} \partial x_{j}}+\operatorname{div}_{L} X_{i} \frac{\partial}{\partial x_{i}}\right)+c^{\prime}
$$

where $c^{\prime}$ is some function. If this operator is carried over to the space $L_{2}(\mu, U)$ through the canonical isomorphism it is of the form

$$
h\left(g^{i j} \frac{\partial^{2}}{\partial x_{i} \partial x_{j}}+\operatorname{div}_{\mu} X_{i} \frac{\partial}{\partial x_{i}}\right)+V
$$

where $V$ is a $C^{\infty}$ function. Since the Laplace-Beltrami operator is given by the coefficient of $h$ in the above expression this completes the proof of theorem 4.1 . 
Remark. In general the solution to Eq. (4.5) need not be unique. If however the manifold is analytic and so are the coefficients $a_{i j}$ then $a_{i j}$ are completely determined by (4.5) if we know their values at one point in $U$. In particular if $a_{i j}=\alpha \cdot g^{i j}$ at any one point in $U$, then $a_{i j}=\alpha g^{i j}$ at all points in $U$. Thus the condition that $C_{U} \neq 0$ at all points in $U$ may be replaced by the analyticity of the manifold $M$ and the coefficients $a_{i j}$ and the equation $a_{i j}=\alpha g^{i j}$ at some point in $U$.

Acknowledgement. The author wishes to thank Professor A. H. TAUB of Berkeley for his criticisms of an earlier version of this paper.

\section{References}

1. Eisenhart, L. P.: Riemannian geometry. Princeton: University Press 1949. 2. MACKEY, G. W.: The mathematical foundations of quantum mechanics. New York: Benjamin 1963.

K. R. Parthasarathy Statistical Laboratory University of Manchester, Oxford Road Manchester 13, Great Britain 